\title{
The Exploration of Computer Aided Translation from the Perspective of Information Technology
}

\author{
Changhong Zhai ${ }^{1, \mathrm{a}^{*}}$ and Xiangxin $\mathrm{Liu}^{2, \mathrm{~b}}$ \\ ${ }^{1}$ School of Foreign Languages, Wuhan Polytechnic University, Wuhan, 430028, China \\ ${ }^{2}$ Department of Public Relations, Wuhan Railway Vocational Technological University, Wuhan, \\ 430325, China \\ a594561792@qq.com \\ *The corresponding author
}

Keywords: Information technology; Computer aided translation (CAT); Translation software; Translation memory technology

\begin{abstract}
Based on the explication of basic principles of the computer aided translation, this essay studies the main functions of the popular computer aided translation software nowadays. What's more, it also builds a way to optimize the process of translation and improve the efficiency and quality of translation by means of translation memory technologies.
\end{abstract}

\section{Introduction}

At present, it has been a urgent desire of people to realize efficient translation between different languages and improve the efficiency to gather information by taking advantage of advanced technologies. The relevant research has been done for several decades and great achievement has been realized. Some commercialized tools have been applied to widely use. The development prospects have become better and better with the unknown research direction at the beginning and the later find of the combination junction of information technologies and translation activities and the breakthrough point of the studies.

\section{The Rationale of Computer Aided Translation}

Computer aided translation is the translation solution based on the translation memory (TM) technology, referred to as CAT and shares some similarities with CAD(Computer Aided Design)technologies.CAT is a kind of translation strategy used by the translator to apply computer programs partly to the translation process, with the thesaurus and corpus stored in the system being its base. The basic working principles of CAT are that the system will divide sentences into separate units such as words and phrases according to its own judgment. And translation suggestions of sentence units will be given according to the lexicon stored in the system, created by the user or stored in the system memory. At the same time, the system will search automatically the same or similar existed translation resources (for example sentences and paragraphs) in the translation memory and reveal the reference translations. The translator can firstly make some adjustments to the sentence units divided by the system (if necessary) and then rearrange the sentence units and accomplish the rearrangement of the information according to the sentence units ' translation suggestions given by the system. And the translator can also rearrange the sentence units according to the reference translation provided by the system or revise it or adopt it directly.CAT differs the old machine translation software in that it is not relied on the automatic computer translation but demands people's anticipation in finishing the whole translation process. CAT leaves all the mechanical and trivial work in transition to computer, which automates the cumbersome manual translation process. The translator needs only to focus on the organization of the translation, which can give full play to advantages of both the translators and computer, improve the translation efficiency and quality to a higher level. 
The greatest advantage of the computer lies in the storage and retrieval of information. By using CAT, large quantities of terminologies can be introduced to reduce manual labor of translators consulting and retrieving the dictionary in the translation process.CAT technologies contain the automatic memory and search mechanism which can store automatically users' translation content. When the user is translating some sentence, the system will search automatically the sentence already translated by the user, if the sentence the user is translating was translated before then the old translation results will be displayed automatically, as for some similar sentences, translation references and suggestions will be given. The database is stored in the LAN server in the network- integrated translation memory system, so translators are able to share the database despite of the remote geographical distance between teammates. While the greatest advantage of the translator is its logical thinking capabilities. Translation is a composite activity which consists of the comprehensive abilities of translators including translator's knowledge, bilingual training, thinking ability and expression ability and many such factors. How to translate a word, a sentence or a passage is the decision made by translators through the common effect of the factors mentioned above and after a series of thinking activities like analysis, induction and reasoning. By applying CAT, translators can shift their main focus on the reorganization of the information, which gives full play to the advantages of people's logical thinking to maintain the integrity of the information transmitted in the translation process and the thoroughness of the information reorganization, thus to obtain the best translation effect.

\section{Main Functions of the CAT Software}

There are many manufacturers developing transportation memory (TM) software in the international community, some famous ones are TRADOS, Transit, SDLX, Dejavu and WordFisher. Some mature ones in the domestic market are like Yaxin CATS computer aided translation system, Yongli ETS aided translation system, etc. Looking at the mature and widely used computer aided translation software nowadays, the following functions can be found mostly:

Program Management. Program Management is to provide batch quantity analysis and pre-translation for the files in need of translation. Batch quantity analysis will usually employ some functions similar to that of corpus retrieval softwares, such as evaluate word frequency of the documents to be translated in order to calculate the vocabulary repetition rate that is relatively high, so users can find out the typical terms from the project. In the whole translation process program management provides support and guidance for the work and ensures the quality of translation effectively. For instance, in the situation of a demanding project which acquires several translators' participation, the glossary can be extracted according to the words frequency statistics. And the unified translation version of the glossary can be decided at the beginning of the project and distributed to the translators. In this way, even if there were some mistakes in the glossary established before, they can be found and corrected easily.

Terminology Management. Terminology management is to provide service to translation process by using database technologies to store and retrieve terminologies effectively. Terminologies are different from entries in a dictionary and terminology management plays a very practical role in the professional translation field. When the translator applies the translation memory system to the translation, all he need to do is to unfold the corresponding terminology table in the memory base tools and then he can use the established standard terminology table(including terminologies in the source text and targeted text).

Transaction Unit Management.Translation unit refers to the matching and storage of a sentence unit of the source language and the target language. Translation unit management should be stored and retrieved in real time in translation. Ida pointed out that the translation process would become more and more dependent on computers with the growing size of the database and improvement of the computer softwares, especially on the occasions like helping the translator to know about applying examples of words. After the translator created the translation memory base, this tool can analyze and make comparisons between the source text and the translation automatically and translates in the unit of one 
sentence after another. After the adjustment and coercion of the translator and the translation version is established, the memory base tools will automatically generate translation memory and build a translation memory base effectively. The memory base will be updated and improved in the future use process. Computer aided translation software generally equips the function of corpus query.In translation, it is possible to quickly analyze and compare the consistency of the content of the source text and the source text by starting the search engine program and looking up in the memory base, and the translator can take the translation version closest to the source text as a reference. With the appearance of more and more the repeated sentences, translation will become much easier. The translation memory also supports the translation memory base based on the network sharing technology, which can be shared by the translators when they work at the same time, so that the online translation fruits can be utilized fully by every translator.

Quality Control. At present, CAT' quality control is in a relatively primary stage which generally includes the spelling check of the target language, grammar check and unified examination of the terms. Many modern translation memory systems can not only help the independent translators to maintain the consistency of terminology, but also help the translation agencies to maintain the consistency of the terms of the large translation teams.

\section{The Exportation of the Construction of Computer Aided Translation}

CAT softwares are improved with the development of the information technologies; some of them achieved great effect in the practice of translations. However, the popularization and the knowledge of CAT softwares should be further increased. And how to make use of these softwares to optimize translation process and improve translation efficiency and quality needs to be further studied.

Thesaurus Construction. Thesaurus is the foundation and basis of CAT software to convert information. The existing CAT software is divided into two categories: some without thesaurus which needs the user to build one before using or accumulate it during translation; some are equipped with thesaurus but are imported directly after the electronic processing of some dictionaries, so the professional level and pertinences are limited. To improve the accuracy of information conversion of CAT software, the user has to set up ahead of time the professional and targeted thesaurus. The differences between thesaurus and dictionaries lie in that the entries in the dictionary may correspond to several meanings while entries in the thesaurus only correspond to one meaning. And the user can make manual efforts to build a thesaurus suited for his own professional features and improve the effect of CAT softwares as soon as possible.. The construction of professional thesaurus plays an effective role in improving the software using effect, if combined with the technical information translation of the project and synchronous arrangement of the professional thesaurus, great effect can be achieved through less investment.

Corpus Accumulation. In translation the CAT softwares retrieve the corpus at anytime and will display some identical or similar translation texts translated before to allow translators to choose. Due to the flexibility of natural language, the probability of occurrence of the same sentence is relatively low in different translation projects. It is only in the same batch of technical information, especially in the case of technical information such as technical manuals, that the probability of the occurrence of the same or similar sentences is higher, and the accumulated corpus can play a certain role. In the use of CAT software, it will memorize and accumulate the corpus automatically.

Translation Project Organization.CAT softwares bring convenience for the organization of translation projects. Before the translation, the documents can be preprocessed including pretranslation the extraction of the terminologies and definitions, the dividing of the documents, etc. Through the extraction of the terminologies and definitions, the standardization and unification of the terms of the translated texts can be easily realized. The content translated by the translator will be transmitted to the database in real time; proofreader can check the translation content in real time, so that the speed of translation proofreading is accelerated. In large translation programs, the CAT softwares enjoy obvious advantages. 


\section{Summary}

The success of the application of information technology in the translation process achieves a breakthrough from the failure of Machine Translation's exploration to the successful application of computer aided translation technology. With the simple memory technology, convenient human-computer interaction mechanism and open thesaurus, memory and terminology databases applied by CAT softwares, it provides an efficient translation platform for professional translators greatly improves the translation environment and improves the efficiency and quality of their translation. Scientific and technical translation is still playing an important role in the scientific and technological information work. The intelligence department should adapt to the development of technology and attach great importance to the introduction and application of CAT technology. The greatest advantage of CAT software is that it has replaced a lot of tedious repetitive manual labor in translation, and at the same time preserves the achievement of many translators to form the memory base, which is consistent with the concept of knowledge management. A large translation memory base of high quality will become valuable knowledge assets of the company.

\section{References}

[1] Zhu Yubin. A Comparative Study of Four Kinds of Common Computer Assisted Translation Software at Home and Abroad [J]. Computer-Assisted Foreign Language Education, 2013(1).

[2] Li Jun. The Application of Computer Assisted Softwares [J].Journal of Language and Literature Studies, 2013(10).

[3] Sun Feiran. Computer Assisted Translation Based on the Era of "Internet +"[J].Automation \& Instrumentation, 2016(5).

[4] Chen Sili. A Study of the Advantages and Limitations of Computer Assisted Translation in the Age of Internet Technology [J]. Electronic Test, 2013(14).

[5] Wang Xin. New Translation Machines in the Age of Big Data [J]. Modern Communication, 2014(10). 